\title{
Pratiques
}

Linguistique, littérature, didactique

137-138 | 2008

La didactique du français

\section{Littératie et didactique de la culture écrite}

Jean-Louis Chiss

\section{(2) OpenEdition}

Journals

Édition électronique

URL : http://journals.openedition.org/pratiques/1158

DOI : 10.4000/pratiques. 1158

ISSN : 2425-2042

\section{Éditeur}

Centre de recherche sur les médiations (CREM)

\section{Édition imprimée}

Date de publication : 15 juin 2008

Pagination : 165-178

\section{Référence électronique}

Jean-Louis Chiss, "Littératie et didactique de la culture écrite », Pratiques [En ligne], 137-138 | 2008, mis en ligne le 15 juin 2008, consulté le 14 novembre 2019. URL : http://journals.openedition.org/ pratiques/1158; DOI : 10.4000/pratiques.1158

(C) Tous droits réservés 


\title{
Littératie et didactique de la culture écrite
}

\author{
Jean-Louis Chiss \\ Université Paris 3 Sorbonne Nouvelle, EA 2288, DILTEC
}

\begin{abstract}
"Pour les pêcheurs de Bouzigues, lire ne change rien dans le fond. C'est peut-être dommage mais il faut bien admettre que la vie académique n'est pas la seule vie possible » (Goody, 2007, p. 240).
\end{abstract}

L'article de Jean-François Halté « Les conditions de production de l'écrit scolaire » et mon article « La grammaire et les textes : deux fonctions de l'écriture » paraissent dans le numéro 11 d'Enjeux (janvier 1987) au moment où se fonde la DFLM (Association pour le Développement de la Recherche en Didactique du Français Langue maternelle) devenue AIRDF il y a quelques années. Après deux colloques généralistes et initiateurs de la constitution du champ en 1981 et 1983 , le colloque de Namur de 1986 prend pour première thématique spécifique la production de textes écrits définie comme « objet crucial» sur le plan des recherches et lieu « stratégique dans la lutte contre l'échec scolaire » (Laurent, 1987, p. 7).

Je me plais à penser aujourd'hui, en prenant pour point de départ de ma réflexion, en guise d'hommage à Jean-François, ces deux interventions, que les directions présentées illustraient les deux focalisations possibles dans ces années 1980 sur l'écrit, points de vue moins divergents que complémentaires, la communication et la culture de l'écrit constituant deux questions essentielles pour la didactique du français (Chiss, 1995/2005). Alors que J.-F. Halté mettait au premier plan des impératifs didactiques le fait de «produire de l'écrit dans une perspective communicationnelle » $(1987$, p. 41) et insistait sur les déterminations communicationnelles des écrits » (ibid., p. 48), je voyais, au même moment, dans la notion de "littératie " un élément central de la recomposition disciplinaire des études sur l'écrit, dans un trajet qui essayait de montrer une certaine compatibilité des débats sur le statut de l'écriture dans la linguistique avec les références anthropologiques ( $c f$., entre autres Chiss et Puech 1997 et Chiss 
1998). Plutôt que d'opposer frontalement, dans les problématiques de l'écriture, un paradigme scolaire-linguistique-cognitif et un paradigme éducatif-socioculturel-communicatif, il me semble que la tension entre communicatif et cognitif (Chiss, 2003) constitue le fil rouge de l'examen des relations entre oral et écrit et du statut de l'écriture en didactique des langues. Le numéro 131/132 de Pratiques sur la littératie et, en particulier l'article d'Yves Reuter, vient, vingt ans après Namur, relancer explicitement dans notre communauté de didacticiens un débat qui n'a en fait jamais cessé et dont je souhaite reprendre ici quelques éléments à partir de ma contribution personnelle à la pertinence de cette notion dans la pensée didactique française/francophone.

\section{La littératie dans le débat sur l'échec scolaire}

Quand j'ai noté en 1995 que la littératie était un concept pour la didactique plutôt que de la didactique, il s'agissait tout autant de repérer les lignes de force d'un contexte favorable à un intérêt pour les contenus rassemblés sous cet intitulé que de commencer à montrer sa force heuristique pour l'élaboration disciplinaire de la didactique du français. On sait que le procès de constitution de cette didactique n'a pu faire abstraction (comment aurait-il pu en être autrement?) de la genèse multiple des « rencontres intellectuelles » et de la manière dont se nouent les intérêts de connaissance. Si Reuter (1997) voit bien que pour Goody la scolarisation est partie intégrante de la littératie, la typologie rétrospective qu'il construit des structures d'accueil de la littératie dans les travaux didactiques ne prend en compte que certains trajets ou en minorise d'autres, par exemple la relation entre linguistique, didactique et littératie.

Avant de procéder à un élargissement de perspectives, il me semble utile de revenir sur le lien entre littératie et compétences en lecture-écriture en rappelant des éléments du contexte des années 1980-1990 où quatre problématiques centrales, qui se recoupent partiellement, favorisent l'entrée de la littératie : l'illettrisme et le corollaire des écrits sociaux (déchiffrer les villes avec les mots, les chiffres et les lettres partout, enseignes et menus, lettres et chèques...) ; 1'écriture et le monde du travail (les écrits professionnels et leur fonction structurante dans la vie de toute entreprise) ; l'immigration et la diversité culturelle des littératies chez les adultes et à l'école ; la persistance des débats généraux sur l'échec scolaire en langue « maternelle », la « maitrise de la langue » (avec ses ambiguïtés) tenant le haut du pavé dans les programmes et curricula de l'école et du collège français. Dans le contexte francophone québécois, qui joue aussi un rôle de diffuseur des travaux anglophones sur la « literacy », un article comme celui de Painchaud et alii (1994) parcourait ces multiples terrains avec le souci de montrer que la littératie fournit un cadre de pensée pour appréhender la situation de la culture de l'écrit dans la société et discuter les nécessités de relever les standards de lecture-écriture.

Les polémiques déjà anciennes sur la «baisse du niveau », les enquêtes internationales aux résultats pour la France aussi médiocres que contestés, les évaluations nationales dans le système éducatif aux diagnostics pertinents mais aux causalités contestables, des rapports officiels approximatifs suscitant des programmes scolaires en perpétuel changement, minorant ou valorisant dans une constante valse-hésitation l'oral ou l'écrit, sacralisant le débat sur les « métho- 
des » de lecture ou le réduisant à la portion congrue, alertent sur la nécessité de reprendre quelques débats de nature épistémologique.

L'actualité résonne toujours des évaluations internationales : 1'enquête PISA (Programme international pour le suivi des acquis des élèves) menée par l'OCDE en 2006 (teste les élèves de 15 ans) place la France au $19^{\mathrm{e}}$ rang; 1 'enquête PIRLS (Progress in International Reading Literacy Study) de 2006, publiée le 28 novembre 2007, qui teste les élèves de 10 ans, classe la France à la $27^{\mathrm{e}}$ place sur 40 pays. On connait la méfiance française face à ces évaluations qui s'est traduite entre autres par l'abandon par la France fin 1995 d'une précédente enquête de l'OCDE sur la littératie ${ }^{(1)}$ ou par les argumentaires de sociologues et statisticiens certes justement attentifs à la complexité des variables, aux biais culturels, mais dessinant néanmoins en creux une certaine « résistance » à la littératie qu'il s'agit précisément d'analyser (Chiss, 2004a). C'est encore, dans l'actualité, le maintien d'explications qui n'expliquent rien. Ainsi, Le Monde du 29/12/07 (p. 10), titre «L'apprentissage de l'histoire et de la géographie dépend étroitement de la maîtrise de la langue », résumant les conclusions de deux études de la direction de l'évaluation, de la prospective et de la performance (DEPP) du ministère de l'éducation nationale sur les acquis des élèves en fin d'école primaire et en fin de collège en histoire, géographie et éducation civique. Tout se passe comme si l'étiquette commode, non fondée théoriquement de « maîtrise de la langue », continuait à dispenser d'une réflexion approfondie sur la compréhension de l'écrit, là où il est question de langage, c'est-à-dire de la complexité des relations entre langue, culture et cognition.

Le recours à la notion de « littératie » permet de replacer nos débats contemporains dans une histoire de la longue durée pour ressaisir les éléments de spécificité que recèle la situation de «scolarisation universelle ». Si le problème des compétences et de leur évaluation est illustré par PIRLS ou PISA aujourd'hui, il se posait en Grèce, en Mésopotamie ou en Chine : «De nos jours encore des questions se posent quant à la façon d'évaluer le degré de littératie et le niveau de compétence en lecture et en écriture à considérer comme « fonctionnel » dans une société donnée » (Goody, 1968a, 2006, p. 26). Le lien entre écriture et école est constamment affirmé par l'anthropologue ${ }^{(2)}$, doublement présent à travers le rôle cognitif de l'écrit dans le développement de l'apprenant et à travers la structuration scripturale des disciplines scolaires et de l'ensemble d'une culture spécifique : «Or les écoles ne sont pas organisées pour accueillir la culture des bergers des Cévennes ou des pêcheurs de la Méditerranée » (Goody, 2007, p. 239). Goody (2007, p. 70) élargit la perspective aux relations entre écriture et apprentissage : «La reproduction génétique est en grande partie autoreproductrice, mais l'apprentissage humain implique des processus génératifs, "apprendre à apprendre", selon 1'expression de Bruner ».

Il ne s'agit pas, chez Goody, d'occulter la réalité de la transmission de certains

(1) La presse a récemment rappelé l'effroi à l'époque devant les types de questions posées : « un gramme de matière grasse contient 9 calories. Quel pourcentage de calories provient des matières grasses totales dans un Big Mac, sachant que ce hamburger, d'un poids total de $215 \mathrm{~g}$, contient 500 calories et $26 \mathrm{~g}$ de matières grasses totales ?»

(2) «L'écriture étant si étroitement associée à l'école, il est inévitable que je revienne sur ce sujet à plusieurs reprises »(Goody, 1994, p. 15). 
savoirs dans les sociétés uniquement régies par l'oralité mais de souligner le rôle démultiplicateur de l'écrit et de l'école dans ce processus ainsi que la différence entre le mode de pensée de l'école et les fonctionnements des socialités familiales et amicales :

« Si, par exemple, nous revenons aux raisons pour lesquelles l'enseignement obligatoire et universel n'a pas permis d'atteindre le niveau de résultats intellectuels, sociaux et politiques qu'attendait James Mill, nous pourrions être amenés à imputer la plus grande part de responsabilité au fossé qui sépare la tradition littératienne prévalant à l'école publique des traditions orales, très différentes, souvent en pleine contradiction avec les précédentes, propres à la famille de l'élève et à ses pairs. Les grandes différences concernant le degré d'exposition des individus à la tradition littératienne créent un fossé dans la société littératienne que la société non littératienne ne connait pas ; ce fossé est celui qui sépare les diverses formes de littératie et de non-littératie. C'est à l'école, cette institution-clé de la société, que ce conflit se manifeste de la façon la plus spectaculaire » (Goody, 1968b, 2006, p. 60).

Alors même que Goody (2007) note une nouvelle fois qu'il n'a pas tant défendu la thèse du « grand partage » entre oral et écrit qu'il a cherché à expliquer ce partage, cette bipartition reste centrale dans les élaborations en didactique, effaçant la complexité du « continuum », minorant les degrés entre littératie « restreinte » et « littératie étendue » pour les sociétés et les individus. L'important est sans doute ici de mesurer le changement de point de vue qui s'est opéré dans la pensée des relations entre oralité et écriture dès lors que les cultures écrites ont cessé d'être minoritaires : «En conséquence, une soi-disant tradition orale qui complète une tradition écrite ne peut pas être assimilée à la tradition orale d'une société sans écriture » (Goody, 2007, p. 47). La volonté justifiée de redonner à l'école une importance cruciale à l'apprentissage du langage oral, de faire droit dans la réflexion didactique à un oral « réflexif» (Chiss, 2003 et pour une illustration parmi d'autres Plane et Garcia-Debanc, 2002), d'examiner les diverses formes de l'oralité dans 1'histoire culturelle de la transmission des connaissances (voir Waquet, 2003), apparaît moins contradictoire avec les « implications » de la littératie que partie prenante de ses «conséquences » dans les sociétés qu'elle a investies en redistribuant les territoires et bouleversant les hiérarchies.

Dans ces conditions, on peut comprendre la logique des réponses que Goody apporte à certains de ses contradicteurs comme Brian Street ${ }^{(3)}$ (Goody, 2007, pp. 21-26) qui lisent ses travaux sur la littératie comme une survalorisation de l'écrit évidemment solidaire d'une dévalorisation des cultures orales, lecture classique des tenants du relativisme culturel, soupçonnant les prétendus sectateurs de l'écrit de « différentialisme ». Dans son approche de la relation entre littératie et didactique, Y. Reuter reprend ce débat dans des termes qui "protègent» Goody tout en alertant sur les modalités de sa réception dans le contexte français en particulier (sur ce point, voir Chiss, 2004a) :

« Cette lecture est sans aucun doute réductrice et étrangère à la pensée de Goo$\mathrm{dy}{ }^{(4)}$, elle me parait cependant sous-jacente à certains de ses emprunts et, au moins pour une partie, explicative de sa fortune réalisant une conjonction entre

(3) Auteur de Literacy in Theory and practice, Cambridge University Press, 1984.

(4) Ce que confirme, si besoin est, la lecture de Goody, 2007 (c'est moi qui précise). 
approches théoriques "modernes" et tradition lettrée, doxa sur-valorisant la culture écrite (ou du moins certaines de ces (?) formes), l'associant à Pensée et Rationalité (uniques), construisant les pratiques populaires sous les formes du manque et du déficit...»(Reuter, 2006, p. 139).

En évoquant, en note, les pamphlets sur la « crise de l'école» pour appuyer sa thèse d'un accueil favorable du concept de littératie dans les milieux lettrés, Reuter me semble commettre une erreur d'analyse que renforce l'acceptation de la dichotomie entre «culture écrite » et «pratiques populaires ». Je prendrais pour ma part l'exact contrepied de ce diagnostic en posant que la « résistance » à la littératie provient essentiellement des tenants de la culture des Lettres qui en critiquent les aspects « fonctionnels », « techniques », les « savoir faire de la vie quotidienne " (équivalent de « literacy » dans les enquêtes internationales) ne faisant pas plus recette dans ces milieux que la culture scientifique et technique. L'orientation textualiste et lettrée de la culture dominante du langage en France où la figure du lecteur se confond avec celle du lettré au point que l'illettrisme restait dépourvu de contraire «positif » jusqu'à la fabrication du néologisme « littérisme » (Journal Officiel du 30 août 2005), se soutient de tout un complexe intellectuel et de ses traductions institutionnelles et mondaines (voir Chiss, 2001). S'il fallait parler en termes de facilité de réception ou de continuité de tradition, on pourrait sans doute suggérer que la rhétorique française de l'écrit s'est mieux accommodée des schémas planification/textualisation/réception (Hayes et Flower) et des « grammaires de texte en général. Dans la culture éducative de 1 'enseignement primaire, au tournant des années 80 , certains spécialistes en lecture-écriture ont trouvé une inspiration chez les psychologues cognitivistes au grand étonnement des linguistes-didacticiens. Les Programmes du primaire de 2002 déferont cette alliance conjoncturelle, les penseurs de l'éducation se tournant vers la littérature, la communauté des lecteurs, l'interprétation, délaissant le cognitivisme pour l'herméneutique : rupture dans la continuité ?

Les croisements et recouvrements entre la littératie et les problématiques didactiques (en particulier ce qui se trouve engagé sous le vocable de « didactique du français »), impliquant cette discussion principielle sur les compétences requises à l'écrit, gagnent à être à la fois réaffirmés et orientés dans les directions suivantes : structuration à l'écrit, par l'écrit, des contenus disciplinaires ; spécificité des systèmes d'écriture ; fonctionnements différenciés des cultures écrites ; continuum décrit par la littératie, et en particulier lien entre littératie et littérature. Ces directions recoupent largement celles de Reuter (2006) avec le souci, comme il l'affirme en conclusion (p. 151) de "prendre au sérieux » Goody et la didactique, mais avec des inflexions qui tiennent aux trajectoires intellectuelles qui ont déterminé la « rencontre » avec la littératie.

\section{Oral, écrit, mémoire}

"La parole est irréversible, soit: on ne peut reprendre un mot, sauf à dire précisément qu'on le reprend. Ici raturer, c'est ajouter; si je veux gommer ce que je viens d'énoncer, je ne puis le faire qu'en montrant la gomme elle-même (je dois dire: "ou plutôt", "je me suis mal exprimé...") ; paradoxalement, c'est la parole, éphémère, qui est indélébile, non l'écriture monumentale. A la parole on ne peut que rajouter une autre parole. Le mouvement correctif et perfectif de la parole est 
le bredouillement, tissage qui s'épuise à se reprendre, chaîne de corrections argumentatives où vient se loger par prédilection la part inconsciente de notre discours (ce n'est pas fortuitement que la psychanalyse est liée à la parole, non à l'écriture : un rêve ne s'écrit pas) : la figure éponyme du parleur, c'est Pénélope » (Barthes, 1971, p. 3)

«Verba volant, scripta manent ». La sagesse populaire a saisi ce qui fut longtemps le fondement d'une différence et l'emblème de fonctionnements sociaux dans les sociétés affectées par le développement de la littératie. Mais voici que Barthes nous alerte sur une possible inversion : les paroles restent, la correction n'annule pas ce qui a été dit, les «verba » ne « volant» pas, ce que confirment l'enregistrement de la parole dès la fin du XIX ${ }^{\mathrm{e}}$ siècle et nos expériences de parleurs ordinaires qui n'oublient pas un mot ou un échange, dans une époque aussi affairée à la recherche de la «parole vive» du témoignage. Ce sont désormais les « scripta» qui ne «manent» plus ${ }^{(5)}$, effacées par un clic sur l'ordinateur, encore plus radicalement qu' avec la gomme ou le «blanc », sans « traces », pourtant si précieuses pour le généticien des textes ou l'écolier retravaillant son «brouillon ». L'effacement désacralise l'écrit d'autant plus que la duplication à l'infini en fait un objet dégradable et non conservable.

Si l'on se place dans une logique qui privilégie la communication (et c'est la question des genres qui est alors primordiale), qu'elle soit différée ou non, les différences entre oral et écrit s'estompent : l'importance du face à face conversationnel de l'oral (avec le rôle régulateur de l'autre, la manifestation de son intérêt, ses demandes de précision...) recule, même si la gestion du temps et la non présence physique continuent à fonctionner comme des contraintes. Mais dès lors que la production écrite s'inscrit dans une logique où domine la signification, alors la nécessité s'impose de choisir ce dont on va parler, d'anticiper sur la compréhension du lecteur, donc de planifier, de décontextualiser l'énonciation pour que le message devienne un produit autonome, lisible, compréhensible, sinon en tous lieux, du moins dans les lieux auxquels il est destiné (école, travail, domicile). Certes certaines situations orales mettent en œuvre du langage décontextualisé : je peux - sans jouer - expliquer la règle d'un jeu ou expliquer un savoir faire sans faire mais l'expérience atteint vite ses limites.

Si la fixation et l'enregistrement ne sont plus l'apanage du mode écrit parce qu'existent des possibilités de manipulation, de reprise, d'effacement (donnant lieu à « brouillons oraux ») au moyen d'appareils enregistreurs de la parole ouvrant sur une véritable « écriture sonore », c'est longtemps la nécessité de fixer les savoirs qui a imposé le recours au mode écrit. On sait que souvent, cette fixation se traduit par une condensation de l'information qui s'accommode mal de l'oral ; cette condensation implique elle-même le recours à des opérations linguistiques comme la nominalisation ${ }^{(6)}$. Mais désormais la spécificité de l'écrit ne se joue pas ou plus sur cette scène-là. En réalité, cette fixation est une forme

(5) Roger Chartier (2005) nous alerte, à propos d'un auteur du XI ${ }^{\mathrm{e}}$ siècle, sur le caractère éphémère de l'écriture sur tablettes de cire, nécessitant la transcription sur parchemin. Les modalités de 1'effacement dépendent de celles de l'inscription...

(6) «Le report de la cotation des actions de la SBS a été décidé à la suite d'une contestation de l'augmentation du capital par les actionnaires » est un énoncé quasi impossible dans l'oral spontané, conversationnel, ne serait-ce qu'à cause de la difficulté de mise en mémoire. 
de constitution pour un univers référentiel dont la complexité informative est très forte et qui demande donc un effort cognitif particulier : plus le matériau informatif à traiter est complexe et plus s'impose la nécessité de l'écriture ou plus précisément sans doute de la scripturalité.

La notion de littératie est arrivée sur le devant de la scène didactique au moment de la prise de conscience de la polysémie, de l'ambiguïté voire de l'insuffisance d'une conception de la communication que l'univers pédagogique assimilait peu ou prou à l'expression. C'est alors la question centrale du traitement et de la mise en forme des savoirs qui a déterminé l'intérêt pour le rôle cognitif, structurant de l'écriture et, plus largement, pour le mode de pensée impliqué par l'écrit. Si la littératie est l'usage de l'écrit dans le cadre d'activités culturelles, les dimensions de cet usage incluent, en particulier dans les institutions éducatives, les formes de la constitution et de l'appropriation des savoirs, les techniques requises pour ce faire et la maitrise des espaces graphiques, l'ensemble de ce que Goody nomme souvent une «technologie de l'intellect».

On reste toujours surpris de constater dans de nombreux travaux en didactique la difficulté à réinscrire débats et préoccupations dans la longue durée de l'histoire ou le décentrement ethnologique. Qu'il s'agisse des espaces graphiques (le tableau noir, l'écran d'ordinateur, le cahier-journal de l'instituteur, le cahier de textes de l'élève), des exercices scolaires (la copie, la récitation), des caractéristiques rhétoriques (digression, répétition), l'entrée par la littératie réévalue et modifie jugements et perceptions. Ainsi par exemple, le lien intrinsèque argumenté par Goody entre canonisation ou codification (de l'alphabet, des tables de multiplication) et mémorisation, ouvre-t-il des perspectives plus heuristiques pour l'école que les déclarations, d'autant plus péremptoires qu'elles ne s'accompagnent d'aucune justification, des sectateurs de l'apprentissage par cœur (voir le retour de la récitation dans les projets de programme de 2008 pour le primaire). De manière générale, la question de la mémoire dans les traditions culturelles (religieuses en particulier) établit des partages toujours mouvants entre oral et écrit ${ }^{(7)}$.

Alors que les cultures orales font l'expérience de mémoires partielles et instables, « ce sont les sociétés de l'écrit qui font proliférer les moyens mnémotechniques, non seulement verbaux, mais aussi spatiaux, comme ceux analysés par Frances Yates dans son célèbre ouvrage The Art of Memory » (Goody, 2007, pp. 77-78) La spécificité de l'écriture est la transmission de mémoire verbatim, la récitation mot à mot; c'est le sens à accorder à cette remarque de Goody

(7) Dans la liturgie catholique, à Cluny, la «memoria », ce sont les noms des défunts qu'on récite à l'office. Ils sont écrits dans le « livre de vie ». La relation entre l' " effet de liste " (Goody) et l' «art de mémoire »se manifeste aussi dans la tradition juive où l'on rappelle le nom hébraïque des défunts qui ne sont pas écrits, le rappel est un appel, avec les risques de l'oubli. Mais la lecture des noms écrits existe aussi, ceux des « exterminés » par exemple le jour consacré à la commémoration de la Shoah (Yadvashem, dénomination du mémorial à Jérusalem, signifie le « lieu des noms »). Le lien de l'écriture à l'oralité se maintient dans la perception même de la Bible :

«L'Ecriture sainte est une lecture sans autorisations. Elle appartient à tous, même aux analphabètes qui ne peuvent que 1'écouter. Rien que 1'écouter? C'est mieux que la lire : les juifs appellent leur corps sacré, l'Ancien Testament, «Mikra », lecture à voix haute. Elle est faite pour être prononcée par des lèvres, une bouche, un son, un souffle, un corps, et pas seulement parcourue par les yeux. La prière juive la plus importante dit «Ecoute Israël», car la prière est une disposition à l'écoute, silence intérieur et environnant, puis prononciation de syllabes » (Erri de Luca, Le Monde des livres, 14 mars 2008, p. 6). 
(2007, p. 43) : «Il est intéressant de noter l'importance de la copie et peut-être aussi de la récitation, c'est-à-dire de l'intériorisation de textes écrits sous une forme précise » (c'est moi qui souligne). On comprend que ce soient les cultures écrites qui réclament ce type de mémorisation et de transmission au mot à mot mais surtout qui permettent la congruence entre cette exigence et la qualité de la prestation orale (interprétation dont la diction est le vecteur) alors que, dans les sociétés orales, « Toute performance est aussi un acte créatif, et il n'y a pas de séparation stricte entre interprète et créateur» (Goody, 2007, p. 73). La récitation scolaire, hâtivement rangée sous l' « oral » est un produit de la culture écrite.

De manière générale, l'insistance justifiée dans les premiers apprentissages de l'écrit sur l'importance de la structuration pré requise du langage oral, sur la conscience phonologique et sa relation à la découverte du principe alphabétique (lui-même par définition inscrit dans la culture de l'écrit) ne saurait masquer les spécificités de l'écrit et d'abord la visibilité sur laquelle insiste Goody dans la lignée d'ailleurs d'une tradition bloomfieldienne (non citée par l'anthropologue) qui enracine la différence oral/écrit dans celle plus large de l'audible et du visible (voir, sur ce point, Anis, Chiss et Puech, 1988). La littératie devient souvent synonyme de «the visible language » (Goody, 2007, p. 237) et la distinction critique pour Goody se fait entre le visuel sur support durable et le non visuel. Cette dimension visible de l'écrit se retrouve en particulier dans l'ontogenèse des signes graphiques à travers le trajet qui mène l'enfant des dessins à la phonétisation (voir le travail de Fereiro et Teberosky, 1979, traduit significativement en anglais sous le titre Literacy before schooling en 1982).

Ce rappel devrait aussi permettre de tirer certaines conséquences pour la didactique de la lecture, en tout cas de contribuer à lever un malentendu persistant dans les débats sur les «méthodes » d'apprentissage. Sans revenir sur les prescriptions hâtives et péremptoires édictées par le précédent ministère et désormais « corrigées » par les nouveaux programmes de 2008, il s'agit de réaffirmer que la discussion théorique, qui a existé dans les années 1970-1980, sur la conception des relations oral/écrit, sur les spécificités de l'écriture ${ }^{(8)}$, discussion argumentée en France par Nina Catach ou Jacques Anis, que même les positions " idéo-visuelles » défendues par des sémiologues et psychologues (voir les travaux de F. Smith par exemple) n'ont pas grand-chose à voir avec la pseudo « méthode globale » brandie comme un épouvantail pour expliquer des carences bien réelles de notre cursus en lecture, celles d'un travail exigeant et continu à l'école primaire sur la compréhension.

En soulignant que l'écriture n'est pas intrinsèquement liée au texte, en mettant en évidence une écriture paradigmatique à fonction structurante qui a exercé en retour une action sur le langage lui-même, avec l'omission des verbes ou le style télégraphique, Goody a contribué à défaire partiellement une relation entre oral et écrit que maintenait la polarisation sur «les « textes continus» (religieux, philosophiques ou littéraires), pourtant tardifs et minoritaires historiquement. Alors que la décontextualisation et l'abstraction étaient déjà à l'œuvre dans l'é-

(8) Goody, dans le cadre de sa vision d'ensemble de la littératie (2007), n'hésite pas, pour sa part, à évoquer, même allusivement (il ne mentionne pas le projet de "grammatologie ») la critique que fait Derrida du «logophonocentrisme »; pour Goody, cette vision derridienne conduit à minimiser les différences entre oral et écrit. Sur ce point, en relation avec la lecture que fait Derrida des positions de Saussure, voir Chiss et Puech (1997, pp. 91-104). 
volution même des systèmes d'écriture ${ }^{(9)}$, c'est l'emploi partiellement décontextualisé du langage dans des contextes formels (les listes pour l'administration ou les tablettes) qui préfigure bien des usages d'une scripturalité précisément non réductible à la textualité et qui investit les cultures éducatives et linguistiques (voir, pour une rapide mise au point, Chiss et Cicurel, 2005, pp .1-9). On voit bien que, par exemple, la digression est constitutive de l'oralité et non de la scripturalité, montrant la différence entre des modes de constitution et de transmission des connaissances, que la répétition ou son absence varie dans les deux canaux en fonction des impératifs de la compréhension, ce qui amène à repérer les caractéristiques d'une «culture de l'écrit » dans son opposition et sa complémentarité à une « culture de l'oral ». Il s'agit là de différences de nature cognitive évidemment non superposables aux variations culturelles des manières de dire et d'écrire dans la diversité des sociétés. Cette ouverture vers la pluralité des cultures écrites, des littératies, l'intérêt, pour la scolarisation, du concept de «plurilittératies » (Moore, 2006) sont des chemins heuristiques qui dépassent le présent propos.

\section{Littératie, construction des savoirs et disciplines scolaires}

Il est évident que le point nodal de jonction entre la notion de littératie et l'école tient dans la constitution et les modes de transmission des disciplines, la codification des savoirs étant à partir d'un certain seuil de quantité et de complexité inconcevable sans le mode écrit. Les historiens de l'écriture, en particulier en Mésopotamie et en Grèce, ont montré la présence de l'écrit au centre du politique, de la vie juridique, le saut qualitatif que représentent l'écriture de la loi, le rapport entre la signature et l'installation irréversible du droit écrit.

Les disciplines scolaires héritent, de multiples façons, des fonctionnements et dispositions des disciplines «savantes» dans des logiques de transposition ou de didactisation sur lesquelles nous ne reviendrons pas ici. L'important est de suggérer les éléments de réflexion que la prise en compte des implications de la littératie permet de réactiver. Dans le domaine de l'histoire, on connait l'enjeu théorique et didactique que constituent les modes de constitution, d'organisation et de présentation des contenus disciplinaires : entre la prévalence du récit d'un côté et la domination des listes de dates, des frises chronologiques ou des tableaux statistiques, c'est la conception même de la discipline qui fait débat, ce que traduisent les discussions entre historiens tout autant que la fabrication des manuels scolaires. En géographie, l'invention des cartes a illustré dans toutes ses dimensions le pouvoir de l'inscription graphique, à la fois écriture et dessin, instrument de mémorisation, de communication et de découverte. La carte impose des contraintes de raisonnement, permet de contrôler, fait découvrir de nouvelles informations ; son graphisme spécifique convoque l'idéogramme (par exemple la grosseur du point noir proportionnelle à la population d'une ville donnée).

La géométrie apparait aussi comme un lieu de prédilection du rapport entre représentation graphique et mise en forme cognitive, alliant la figure à la démonstration et donc impliquant l'argumentation écrite. Si ce lien du graphique aux mathématiques est objectivation de l'espace dans la géométrie, il est, en algèbre,

(9) «Les langues écrites doivent sélectionner l'une des nombreuses formes de parole pour établir un standard» (Goody, 2007, p. 44). 
accès à la formalisation par l'écriture symbolique. Les enseignants connaissent la nécessité de travailler en classe sur l'ordre de l'écriture (alignement des opérations par exemple), de faire acquérir la graphie et la lecture des nombres, tous les codages spécifiques de la «langue» véhiculaire des mathématiques (avec ses lettres, ses chiffres et ses signes divers). Sans doute une acception juste de la « numeracy » doit-elle intégrer l'ensemble de ces éléments, ce qui, soit dit en passant, permet de revisiter la « simplicité » polémique du retour aux « fondamentaux » que sont les savoirs « lire, écrire et compter». Aurions-nous oublié l'importance de ces «modes de pensée fondamentaux » (déductif, expérimental, réflexif, critique) dont l'élaboration, selon les termes du rapport Bourdieu/Gros (1989), était une des missions essentielles de l'école?

Les disciplines et les mises en œuvre «technologiques» qu'elles requièrent ne traitent évidemment pas du matériau déjà-là, pré-formaté. Goody (2007) insiste fortement sur le fait que la grammaire comme le dictionnaire organisent et réorganisent le matériau langagier pour les besoins de sa mise en forme scientifique ; il ajoute cette donnée essentielle que ces instruments deviennent des «modèles » pour les locuteurs des langues naturelles, dès lors que se sont opérés ces processus de grammatisation et de lexicalisation ${ }^{(10)}$. L'hypothèse classique de Sapir et Whorf sur les langues comme « visions du monde » se lit aussi dans la proposition littératienne selon laquelle la " façon de segmenter la nature est fonctionnellement liée à la grammaire » (Goody, 1968b, 2006, p. 64). C'est, à travers l'une des orientations phares de la littératie, à savoir la différence entre textualité et scripturalité, qu'on peut appréhender le dictionnaire et les difficultés liées à son maniement : de la maîtrise de l'ordre alphabétique à la disposition typographique des « entrées ", aux regroupements et dégroupements qui engagent une compétence lexicologique (homonymie ou polysémie) sans compter le métalangage associé à la fonction documentaire de l'écrit, le trajet de l'utilisateur du dictionnaire vers une forme d'expertise est parsemé d'embûches.

La grammaire m'était apparue (Chiss, 1987) particulièrement exemplaire du triple mouvement impliqué par la problématique de la littératie : d'une part, la relation entre l'invention de l'écriture et la grammatisation des langues est incontournable; d'autre part, la grammaire se donne, dès son origine, comme une activité de classification, ce que montrent les typologies des «parties du discours » ou, plus tard des catégories grammaticales; enfin, la grammaire dite « moderne » accentue massivement les fonctions représentative et symbolisatrice de l'écriture. La "grammaire textuelle » n'est pas en reste sur ce plan et l'accusation, répétée à l'envi, de "formalisme " trouverait ici à s'argumenter couplée au reproche d' " applicationisme » adressé aux " arbres » (indicateurs syntagmatiques) ou aux schémas narratifs. Dans une didactique du français encore en gestation qui avait mis au premier plan de ses préoccupations la production d'écrits (voir supra), où la question de savoir quels effets avait ou n'avait pas le travail grammatical sur la production textuelle dans un débat général sur les « fonctions » de la grammaire et des activités métalinguistiques, il pouvait

(10) «La même chose est vraie des dictionnaires. Les mots ont partout des significations. Mais les dictionnaires ne vous apprennent pas seulement l'orthographe; ils déclinent les significations sur un mode standardisé, "les définitions de dictionnaire", qui deviennent ensuite la norme et le point de départ de discussions. Combien de discussions universitaires commencent par des expressions du type "le Littré [ou le Robert] définit X comme..."?»(Goody, 2007, p. 208). 
être heuristique de noter la différence fondamentale entre les dimensions cognitives et langagières impliquées par l'élaboration textuelle et la réflexion grammaticale.

Quels que soient les degrés de recours à la planification et les représentations de niveau macrostructurel, la production d'un texte continu est d'abord un phénomène de linéarisation se déployant sur l'axe syntagmatique du langage, la gestion d'une continuité, cohésion, cohérence, progression avec traitement de reprises et d'anticipations. Face à cette posture, à cette expérience de scripteur, le travail du grammairien, apprenti ou expert, est essentiellement celui d'une mise en forme graphique à travers des modes de représentation diversifiés. Ici prévalent l'objectivation et la codification, là, l'intégration de schèmes pratiques, d'un « habitus » qui a partie liée, en quelque manière, avec l'imprévisible. On peut comprendre que l'expression écrite n'ait pas fait l'objet d'un apprentissage systématique au sens d'une progression régie par des règles inhérentes à celles d'une matière elle-même codifiée. Exercice d' « intégration » et de validation de connaissances acquises dans les disciplines régulées (grammaire, orthographe...), elle apparaissait comme une mise à l'épreuve ; quand s'affirmait une spécificité, les démarches scolaires proposaient des stéréotypes, des "patrons » à imiter (à la manière de...), dans une logique d'artisanat, qui s'étend historiquement des classiques « arts d'écrire » aux modernes « ateliers d'écriture ». Dans l'optique de la littératie, la dichotomie codification $v s$ « sens pratique » (terme de Goody) n'oppose pas tant (et pas seulement) l'oral et l'écrit qu'elle ne traverse l'écrit et ses usages, dessinant un continuum où l'individualisation le dispute à la conformité, de l'exercice canonique de grammaire au « texte libre », manière de s'interroger sur les disciplines scolaires et d'entrer aussi dans la relation entre la culture de l'écrit et ce qu'on nomme « littérature ».

\section{Littératie, littérature et lecture(s)}

Dans une acception large mais non plastique de la littératie, en suivant l'un des parcours du concept, de la connaissance des systèmes d'écriture à la construction des discours, j'ai suggéré (Chiss, 1998) que la définition de Goody (en particulier 1994) des « littératures orales » en Afrique mais aussi en Inde comme «formes verbales standardisées » ouvrait sur une approche anthropologique qui importe à notre réflexion générale sur la littérature et son enseignement. La relation entre le fait même de l'écriture et l'émergence de formes poétiques (l'exemple du sonnet) ou narratives ${ }^{(11)}$ (l'épopée comme les « contes populaires ») déplace, subvertit les frontières entre oral et écrit (voir la discussion dans Goody, 1994 sur l'origine écrite de l'Illiade et sa transmission orale) et ce que nous appelons, nous aujourd'hui, la « littérature » (l'emblématique Don Quichotte) laisse entendre l'écho de la question littératie $v s$ cultures orales ${ }^{(12)}$.

(11) «En conclusion, la forme narrative elle-même, la narration au sens prototypique, est souvent vue comme caractéristique de l'oral; c'est cependant un genre moins courant qu' on ne le pense souvent, et c'est dans les cultures qui commencent à subir l'influence de l'écriture qu'elle se développe » (Goody, 2007, p. 125).

(12) Dans l'optique historienne de Roger Chartier, la littérature apparait comme miroir, mise en abyme et laboratoire d'une culture de l'écrit qui implique supports, techniques et pratiques 
La standardisation des formes verbales est la version technique de la canonisation, processus qui affecte textes religieux et profanes : « la littérature entre dans cette catégorie »(Goody, 2007, p. 192). Si on ne peut pas exclure toute forme de canonisation dans les cultures orales, «l'écriture fait changer non seulement l'orthodoxie, mais la notion d'orthodoxie, et, je crois, celles de vérité et d'identité, ou plutôt la perception de la vérité et de l'identité » (ibid. p. 91). A partir de là, on comprend le paradoxe apparent qui consiste en ce que le développement de l'écriture puisse favoriser les ajouts, compléments, critiques et interprétations multiples du texte réputé canonique. La question des oppositions entre la lecture « littérale » des « textes fondateurs » (la Bible, le Coran) et leur lecture assortie des commentaires qu'ils ont suscités, lecture de «lectures », pose le problème du rapport entre vérité et historicité.

On voit bien que, mutatis mutandis, le canon profane littéraire se trouve affecté par ce dilemme. Mais précisément, parce que l'historicité d'un texte n'est ni la réduction aux conditions de production de ce texte ni le relativisme interprétatif de toutes ses « lectures », parce que la relative immutabilité du texte en fait un référent ou un garant, il apparait pertinent de réfuter à l'instar de Goody (2007, p. 29), des thèses herméneutiques radicales comme celle de Brian Street (op. cit., supra) selon laquelle le sens d'un texte dépendrait entièrement de la manière dont on a appris à l'interpréter. Si l'on accepte un saut dans notre actualité didactique, c'est en relation avec les travaux de Goody et de David Olson (voir Chiss, 2004b) que j'ai analysé la montée en puissance du paradigme interprétatif à propos de la « lecture littéraire » au cycle 3 de l'école primaire dans les programmes de 2002. Le débat entre compréhension et interprétation ouvrait conjointement à des conceptions de la lecture et de la littérature et il m'avait semblé fondé de conclure par la mise en exergue du protocole d'Olson (1998, p. 310) : «Les interprétations d'un texte sont sujettes à la même diversité d'attitudes, de l'hypothèse à la croyance, et elles sont révisables sur la base des données. Penser le texte exige du lecteur qu'il apprenne comment on peut interpréter un texte de différentes manières, et qu'il détermine et mette en œuvre l'interprétation la plus pertinente, en fonction des indices que le texte lui propose. La lecture critique consiste à reconnaître qu'un texte peut être compris de plusieurs façons, puis d'en tirer les conséquences en fonction des preuves apportées par le texte ».

En fait, c'est sur le fonds général d'une tension inhérente à la culture de l'écrit entre codification et individualisation, orthodoxie et critique ${ }^{(13)}$, que s'appréhendent l'ensemble de ces considérations. Goody (1968b, 2006, p. 62), dans un passage où il évoque Platon et Tolstoï, montre en quoi l'écriture encourage la re-

de lecture/écriture. A partir du même Don Quichotte, 1'analyse montre à la fois le caractère instable des textes et la capacité de l'imprimé à « donner dignité et pérennité aux créations poétiques » (2005, p. 96). C'est aussi la question classique de la critique littéraire contemporaine qui se trouve revisitée : dans Don Quichotte, « l'effet de réel [...] provient en premier lieu des échanges permanents noués entre la fiction et les conditions techniques ou littéraires qui en gouvernent la composition - aux deux sens du mot - esthétique et typographique » (2005, p. 73) : tout ensemble «la matérialité du texte et la textualité du livre » (2005 p. 57).

(13) « La force hégémonique du canon ou de l'idéologie dominante ne va pas sans être parfois remise en question dans le monde de l'écrit. Son pouvoir n'est pas toujours exercé par les autorités politiques, pas plus qu'il ne leur est réservé. L'écriture peut aussi s'utiliser dans un but révolutionnaire ou critique, elle peut servir la contre-culture » (Goody, 2007, p. 191). 
lation au «privé » et met en jeu, au fond, des conceptions de l'individuation : «Il est probable que d'autres facteurs ont contribué à la prise de conscience de ce phénomène d'individualisation, mais il reste que l'acte d'écrire (en particulier si l'écriture est de type simple et cursif) a joué un rôle de premier plan. Le fait d'écrire en donnant aux mots une forme physique et en les mettant à disposition en même temps que leurs signifiés pour une analyse bien plus approfondie et minutieuse que l'oral ne l'autorise, encourage la réflexion privée. Le journal intime ou la confession permet à l'individu d'objectiver son vécu et lui donne un pouvoir de contrôle sur les transmutations subies par le souvenir sous l'influence des événements ultérieurs ».

Alors même que les positions bien connues de Platon privilégient la méfiance vis-à-vis de l'écriture, source d'amnésie et obstacle à la vérité, seulement accessible par la dialectique, Goody (1968b, 2006, p. 63) rapproche l'esprit des dialogues platoniciens et celui du roman : «Les deux genres d'écrits sont l'expression d'une aspiration intellectuelle caractéristique de la culture littératienne et ils présentent le processus par lequel l'individu, de manière plus ou moins consciente, avec plus ou moins de liberté personnelle, fait des choix, exprime des refus ou procède à des adaptations parmi les postures et les idées contradictoires que lui propose sa culture ». On le lit : ce trajet de la matérialité de l'écrit à une pensée de la littérature est, parmi d'autres, celui que dessine la littératie ; il croise d'autres parcours : du déchiffreur à l'herméneute, de la découverte de la lettre au traitement informatisé du texte... Tous ces chemins pourraient aussi mener à une reconfiguration des problèmes d'apprentissage et d'enseignement de la lecture/écriture dans une didactique de la culture écrite.

\section{Bibliographie}

Anis, J., Chiss, J.-L., PueCH, C. (1988) : L'écriture. Théories et descriptions, Bruxelles : De Boeck Université.

BARTHES, R. (1971) : «Ecrivains, Intellectuels, Professeurs », Tel Quel n 147, repris dans Euvres complètes, tome 2 (1994), Paris : Seuil, p. 1194-1210.

CHARTIER, R. (2005) : Inscrire et effacer. Culture écrite et littérature (XI ${ }^{e}$-XVIII ${ }^{e}$ siècle), Paris : Gallimard, Seuil.

CHISS, J.-L. (1987) : « La grammaire et les textes : deux fonctions de l'écriture », Enjeux $\mathrm{n}^{\circ} 11$, pp. 138-144.

— (1998) : "Plurivocité de l'écriture et "Literacy” », in J.-G. Lapacherie, éd, Propriétés de l'écriture, op. cit. $\mathrm{n}^{\circ}$ 10, pp. 89-94. Pau : Presses Universitaires de Pau.

- (2001) : « La coupure langue/littérature et la discipline français », in A. Boissinot et alii éd, Littérature et sciences humaines. Cergy-Pontoise et Paris : Les Belles Lettres, p. 149-160.

- (2003) : «Le couple oral/écrit et la tension entre communicatif et cognitif », Didactiques de l'oral, « Les Actes de la DESCO », CRDP de Basse Normandie, pp. 13-21. 
— (2004a) : «La littératie : quelques enjeux d'une réception dans le contexte éducatif et culturel français », C. Barré-De-Miniac et alii, La Littéracie. Conceptions théoriques et pratiques d'enseignement de la lecture-écriture, Paris : L'Harmattan, pp. 43-52.

- (2004b) : «Comprendre et interpréter : réflexions sur la lecture littéraire au cycle 3 ", Nouveaux regards sur la lecture, Observatoire national de la lecture. CNDP et Savoir Livre, pp. 45-57.

— (2005) [1995] : « Sciences du langage : le retour», Chiss, J.-L., David, J., Reuter, Y. (éds), Didactique du français. Fondements d'une discipline, Bruxelles : de Boeck, pp. 79-94.

Chiss, J.-L., CiCUREL, F. (2005) : « Cultures linguistiques, éducatives et didactiques », Beacco J.-C., Chiss, J.-L., Cicurel, F., Véronique, D. (éds), Les cultures éducatives et linguistiques dans l'enseignement des langues, Paris : PUF, pp. 1-9.

ChISs, J.-L., PUECH, C. (1997) : Fondations de la linguistique. Etudes d'histoire et d'épistémologie. Bruxelles : Duculot, $220 \mathrm{p}$.

FERreiro, E., TEBEROVSKY, A. (1979) : Los sistemas de escritura en el desarrollo del nino, Mexico DF : Siglo Veintiuno Editors.

Goody, J. (1968a, 2006) : «La technologie de l'intellect», Pratiques n 131/132, pp. 7-30.

- $(1968 b, 2006):$ «Les conséquences de la littératie », Pratiques $\mathrm{n}^{\circ}$ 131/132, pp. 31-68.

- (1994): Entre l'oralité et l'écriture [traduction française de The interface between the written and the oral, Cambridge University Press, 1993], Paris : PUF.

- (2007) : Pouvoirs et savoirs de l'écrit, Paris : La Dispute.

HALTÉ, J.-F. (1987) : «Les conditions de production de l'écrit scolaire », Enjeux $\mathrm{n}^{\circ} 11, \mathrm{p} .40-48$.

LAurent, J.-P. (1987) : « Présentation du colloque », Chiss, J.-L., Laurent, J.-P., Meyer, J.-C., Romian, H., Schneuwly, B. éds, Apprendre/Enseigner à produire des textes écrits, Bruxelles et Paris : De Boeck Université et Editions Universitaires, pp. 5-9.

Moore, D. (2006) : Plurilinguismes et école, Paris : Didier.

OLson, D. (1998) : L'univers de l'écrit. Comment la culture écrite donne forme à la pensée [traduction française de The World on Paper : the Conceptual and Cognitive Implications of Writing and Reading, Cambridge University Press, 1994], Paris : Retz.

PAINCHAUD, G. et alii (1994) : «Diversité culturelle et littératie », Repères. Essais en éducation $\mathrm{n}^{\circ} 15$, Université de Montréal, pp.77-94.

Plane, S., GARCIA-DEBANC, C. (2002) : L'oral pour apprendre, Paris : Hatier. PRATIQUES n ${ }^{\circ}$ 131-132 (2006) : «La littératie. Autour de Jack Goody ».

REUTER, Y. (2006) : «A propos des usages de Goody en didactique. Eléments d'analyse et de discussion ", Pratiques n ${ }^{\circ}$ 131/132, pp. 131-154.

WAQuet, F. (2003) : Parler comme un livre. L'oralité et le savoir (XVI $-X X^{e}$ siècle), Paris : Albin Michel. 